\title{
Estudio preliminar de la avifauna en el campus de la Universidad de la Amazonia, en Florencia, Caquetá, Colombia
}

\author{
Preliminary study of the avifauna in the campus of the \\ Amazonia University, in Florencia, Caquetá, Colombia
}

\author{
Jorge Luis Peña-Núñez*, Amny Fabinela Claros-Morales*
}

\begin{abstract}
Resumen
Introducción: El desarrollo urbanístico representa una amenaza latente para la biodiversidad; sin embargo, zonas como los campus universitarios pueden disminuir el impacto negativo de la urbanización al consolidarse como islas verdes. Objetivo: El presente estudio buscó determinar la diversidad de aves presente en el campus de la Universidad de la Amazonia. Metodología: Se empleó la metodología de conteo por puntos. Resultados: Se obtuvo un total de 363 registros de aves pertenecientes a 14 órdenes, 27 familias y 62 especies. El orden Passeriformes presentó la mayor riqueza con $37(59,6 \%)$ especies, la familia Tyrannidae con $12(19,3 \%)$ especies y Thraupidae con $7(11,2 \%)$ especies. Las especies con las mayores frecuencias de observación fueron Turdus ignobilis (26, 7,1\%), Thraupis episcopus (24, 6,6\%) y Pitangus sulphuratus (23, 6,3\%). El grupo trófico más representativo fue el insectívoro (43,5\%). Se hallaron siete especies categorizadas en los apéndices II y III del CITES, seis especies de aves congregarías y trece especies de aves migratorias. Conclusión: La diversidad de aves hallada en el campus sugeriría que estas áreas representan una fuente importante de recursos como alimento o zonas de anidación, atractivos para las poblaciones de aves residentes y migratorias que habitan el área urbana del municipio de Florencia.
\end{abstract}

Palabras clave: Aves, Campus universitario, Piedemonte Amazónico, Urbanismo.

\begin{abstract}
Introduction: The urban development represents a latent threat for biodiversity, although areas such as university campus can reduce the negative impact of urbanization trough keeping a multipurpose area of green islands. Objetive: The current study looked for defining the diversity of birds present in the campus of the Amazonia University. Methodology: Using for it the point count methodology. Results: Obtaining a total of 363 registers of birds which belong to fourteen orders, 27 families and 62 species. The order Passeriformes had the biggest wealth with $37(59,6 \%)$ species, followed by the Tyrannidae family with 12 $(19,3 \%)$ species and Thraupidae with $7(11,2 \%)$ species. The species with the most frequency of observation were Turdus ignobilis (26, 7,1\%), Thraupis episcopus (24, 6,6\%) and Pitangus sulphuratus $(23,6,3 \%)$. The most representative trophic guild was the insectivorous $(43,5 \%)$. There were found seven species categorized in the appendixes II and III of the CITES, six species of congregations and thirteen species of migratory. Conclusion: The diversity of birds found in the campus would suggest that this areas represent an important source of resources such as food or nesting areas, attractive for the community of resident and migratory birds that inhabit in the urban area of Florencia city.
\end{abstract}

Keywords: Amazonian Foothills, Birds, University campus, Urban planning.

\section{Introducción}

El Piedemonte Amazónico es considerado como una de las áreas más biodiversas del país (Hernández-Camacho en Halffter 1992, Ruiz et al. 2007) sin embargo, esta alta diversidad ha sido afectada drásticamente durante el último siglo debido a procesos como la colonización, el crecimiento urbano, la expansión de la frontera agrícola y el establecimiento de cultivos ilícitos entre otros (Vargas et al. 2005,

\footnotetext{
Programa de Biología, Facultad de Ciencias Básicas, Universidad de la Amazonia, Florencia, Caquetá, Colombia. e-mail: jlmontano87@gmail.com_amnyfamo@gmail.com

Fecha recepción: Septiembre 2, $2015 \quad$ Fecha aprobación: Noviembre 24, 2015

Editor Asociado: Jiménez AM.
}

(c) Rev. Biodivers. Neotrop. 2016; 6 (1): 85-92 
Ruiz et al. 2007, Díaz-Cháux y Velásquez-Valencia 2009b, Velásquez-Valencia 2009).

Entre estos procesos, el desarrollo urbanístico ha sido una de las principales causas de cambio en el paisaje y representa una amenaza latente para la biodiversidad (Clergeau et al. 2001, Marín 2005, Rivera 2006). Este fenómeno aumenta la fragmentación del hábitat y provoca diferentes grados de amenaza tanto a especies residentes como migratorias (Ortega-Álvarez y Macgregor-Fors 2011). La velocidad con que avanzan estos procesos de desarrollo en asocio con la modificación de los ecosistemas naturales, hace necesario ampliar el conocimiento de la avifauna en los ecosistemas urbanos (Marín 2005).

Algunos autores han establecido que la diversidad de la vegetación y el volumen del follaje en áreas urbanas o la presencia de vegetación nativa, se asocian con una alta riqueza y abundancia de aves (Rivera 2006). Otros han encontrado que esta alta diversidad también se relaciona con ecosistemas que presentan alto grado de intervención (Naranjo y Estela 1999, Angarita 2002, Rivera 2006, Muñoz et al. 2007, Velásquez-Valencia 2009). En el caso de las ciudades, existe una alta disponibilidad de recursos necesarios para la alimentación y reproducción de las aves, que las ha hecho atractivas para el establecimiento de sus poblaciones (Muñoz et al. 2007, Díaz-Cháux y Velásquez-Valencia 2009a).

Estos ecosistemas son un mosaico de ambientes como jardines, bosques, rastrojos, pastizales y edificaciones, donde cada uno por separado o la mezcla de algunos de ellos representan hábitats importantes para las aves (Rivera 2006, Muñoz et al. 2007).

Entre los hábitats urbanos que mantienen una disponibilidad importante de recursos se encuentran los campus universitarios, al estar aislados de la intervención que sufren las áreas circundantes se hacen atractivos para la avifauna local (Muñoz et al. 2007). Estas zonas actúan como corredores biológicos, sitios de arribo para aves migratorias o fuente temporal de alimento y refugio, gracias a que en ellas es común encontrar parches de vegetación y áreas arboladas que además amortiguan los efectos negativos de la urbanización sobre la avifauna (Marín 2005).

Teniendo en cuenta lo anterior, en esta investigación se plantea determinar la riqueza y diversidad de aves presentes en el campus de la Universidad de la Amazonia, con el fin de establecer su importancia en la conservación de la ornitofauna urbana presente en el municipio de Florencia, Caquetá.

\section{Metodología}

Área de estudio. El campus de la Universidad de la Amazonia presenta dos sedes (centro y porvenir) localizadas al interior del perímetro urbano del municipio de Florencia, Caquetá, en el Piedemonte Amazónico colombiano, a una altura de $242 \mathrm{msnm}$. El clima local es cálido-muy húmedo, con un promedio de $3700 \mathrm{~mm}$ de precipitación anual. Presenta una temporada seca de diciembre a febrero y una temporada de lluvias de abril a octubre. La temperatura promedio es de $26,1^{\circ} \mathrm{C}$ y la humedad relativa oscila entre $69 \%$ y $86 \%$, siendo mayor en los meses de junio y julio y menor en el primer trimestre del año (IDEAM 2015).

Método de muestreo. Se realizaron cuatro salidas de campo durante el mes de abril de 2011, de las cuales dos fueron a la sede centro y dos a la sede porvenir (Figura 1). Los puntos se establecieron en cuatro diferentes sectores por sede, de manera que abarcaran la mayor parte del área ocupada por las mismas y considerando un radio de observación de $50 \mathrm{~m}$. Los horarios de observación se distribuyeron en dos jornadas, en la mañana desde las 6 hasta las 10 horas y en la tarde desde las 16 hasta las 18 horas, siguiendo la metodología propuesta por Villareal et al. (2006). Se tomaron datos cualitativos de presencia-ausencia de las especies en los puntos. La vegetación de los puntos de observación se describe a continuación.

\section{Sede Porvenir (SP):}

Edificio de profesores (EPSP): presenta amplias zonas de prados con árboles dispersos. Las especies representativas de la zona son: Zygia longifolia, Inga minutula, Guarea guidonia, Cecropia engleriana, Sysygium malaccense, Pouteria caimito, Spathodea campanulata, Trichantera gigantea, Caryota urens, Elaeis guineensis y Heliconia hirsuta.

Tertulia (TSP): presenta pocas zonas de prados, pero abundantes árboles. Sobresalen especies como: Z. longifolia, Bauhinia aculeata, S. malaccense, Cocos nucifera, Licania tomentosa, Senna multijuga, Swinglea glutinosa, Carludovica palmata y Heliconia stricta.

Cancha de fútbol (CFSP): predomina la vegetación herbácea en especial gramíneas y en los alrededores se destacan especies arbóreas como: Z. longi- 


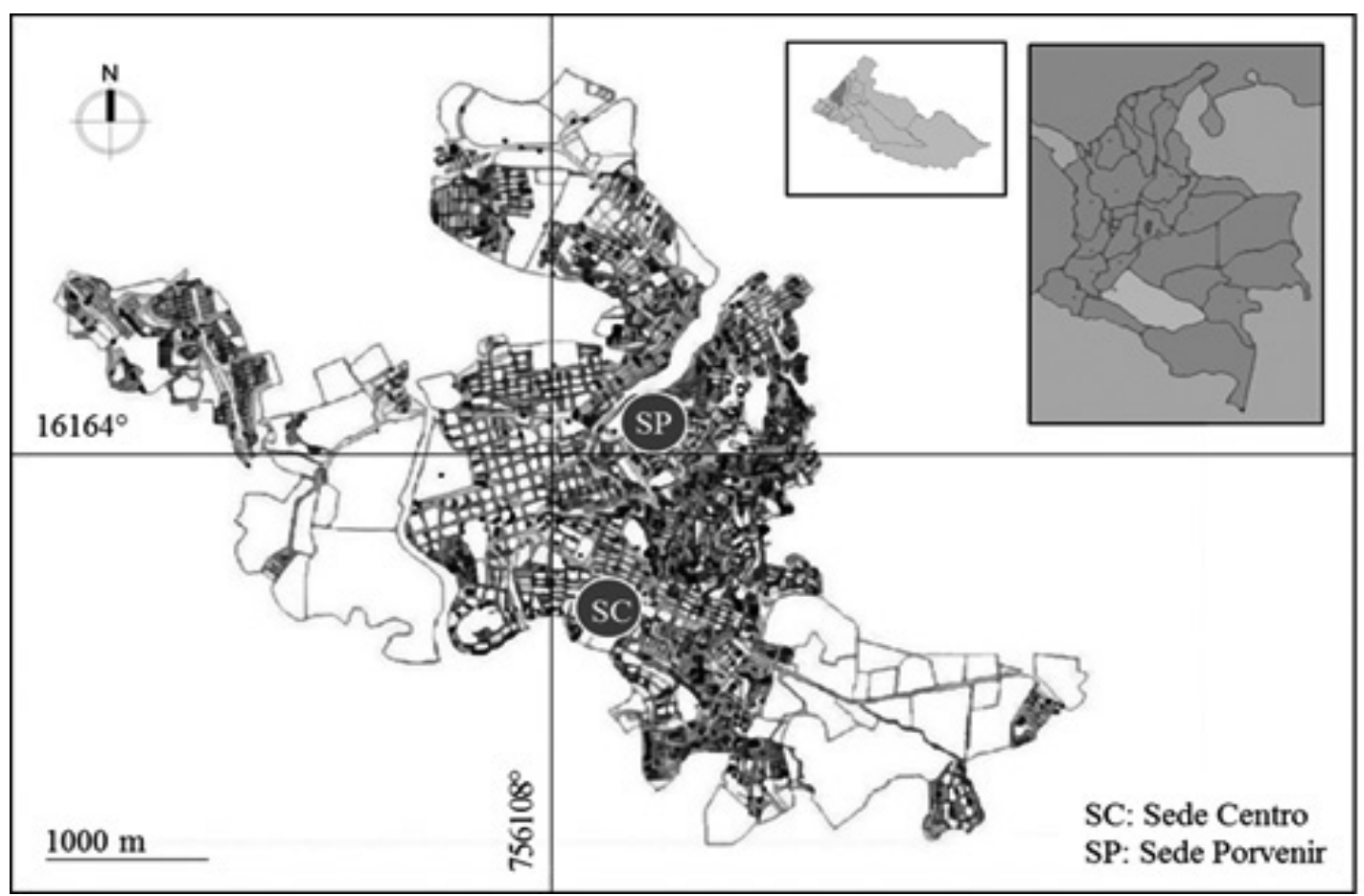

Figura 1. Localización del campus de la Universidad de la Amazonia en Florencia, Caquetá, Colombia.

folia, S. malaccense, Erhytrina fusca y E. guineensis.

Auditorio (ASP): esta área presenta amplias zonas de prados y abundante vegetación arbórea con especies como: Z. longifolia, S. malaccense, Pourouma cecropifolia., Tabebuia rosea, Hevea brasiliensis, Gmelina arbórea, Piptocoma discolor, Ficus sp., T. gigantea, Enterolobium cyclocarpum, Gliricidia sepium, Andira inermis y Caryodendron orinocense.

\section{Sede Centro (SC):}

Cancha de futbol (CFSC): la cancha presenta un sustrato de arena en cuyos alrededores se observan grandes palmas de E. guineensis y Mauritia flexuosa, un rodal de Guadua angustifolia y árboles de G. guidonia, Z. longifolia, Mangifera indica, S. malaccense. A. inermis y Ficus sp.

Araceario (ARSC): la vegetación es densa y predominan especies de aráceas de géneros como: Anturium, Philodendron, Dracontium, Monstera y demás; sobresalen también algunos árboles como: $G$. guidonia, Artocarpus altilis, Erhytrina ulei, Ficus sp. y algunas especies de sotobosque como Carludovica palmata, Piper aduncum, Costus scaber y Heliconia rostrata.

Cycadales (CYSC): se caracteriza por presentar una cobertura herbácea predominante con especies arbóreas como: Psidium guajava, E. ulei, C. engle- riana, E. guinensis, $M$. indica, Ficus sp. y P. discolor. Jardín botánico (JBSC): presenta una predominancia de arbustos y árboles donde sobresalen especies como: P. discolor, Ochroma pyramidale, I. minutula, G. guidonia, A. inermis, C. engleriana, E. guinnensis, Aegiphila sp., Cupania cinerea, M. indica, P. aduncum, C. scaber, C. palmata y H. rostrata.

En cada punto se registraron todas las especies de aves observadas durante 20 minutos, utilizando prismáticos Bushnell 10x42, y para su determinación se consultó bibliografía especializada (Hilty y Brown 2001, Restall et al. 2007, McMullan et al. 2011, Remsen et al. 2015). Las especies fueron categorizadas de acuerdo con los gremios tróficos propuestos por Stiles y Skutch (2003), además se determinaron las especies migratorias, congregarías, con comercio restringido y amenazadas (Renjifo et al. 2002, Murillo-Pacheco 2005, CITES 2015, Naranjo et al. 2012.

Análisis de datos. La representatividad del muestreo se determinó mediante la curva de acumulación de especies obtenida en el programa EstimateS versión 9.1.0 (Colwell et al. 2012), utilizando los estimadores no paramétricos de Sobs (Mao Tau), Chao 1 y 2. Para el análisis de diversidad se utilizó el programa PAST versión 3.04 (Hammer et al. 2001), con el cual se analizó la riqueza mediante el índice 
de Margalef, la dominancia con el índice de Simpson (D), la diversidad con el índice de Shannon (H) y la equidad con el índice de Equitatibilidad (J).

\section{Resultados}

Se obtuvo un total de 363 registros de aves pertenecientes a 14 órdenes, 27 familias y 62 especies. El orden Passeriformes presentó la mayor riqueza (59,6\%), seguido por Apodiformes y Pelecaniformes (6,4\%), Columbiformes (4,8\%), Caprimulgiformes, Cathartiformes, Piciformes y Psittaciformes (3,2\%) y Accipitriformes, Charadriiformes, Coraciiformes, Cuculiformes, Galliformes y Suliformes (1,6\%) (Figura 2). Las dos familias con mayor riqueza fueron

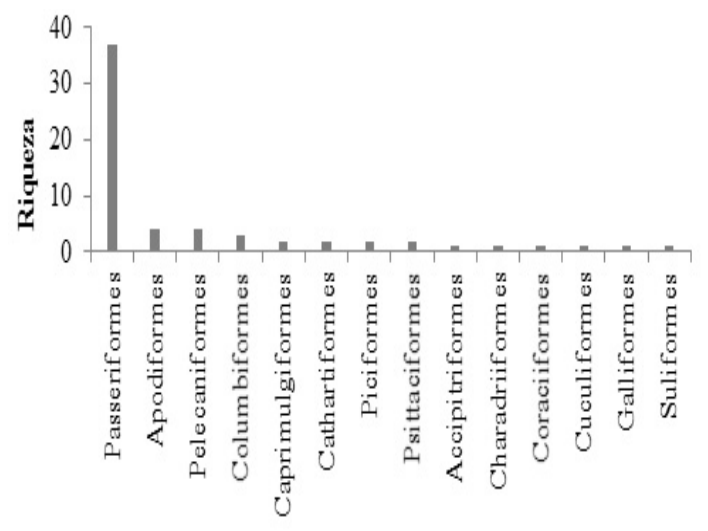

Figura 2. Distribución de la riqueza específica para los órdenes de la comunidad de aves presente en el campus de la Universidad de la Amazonia, en Florencia, Caquetá, Colombia.

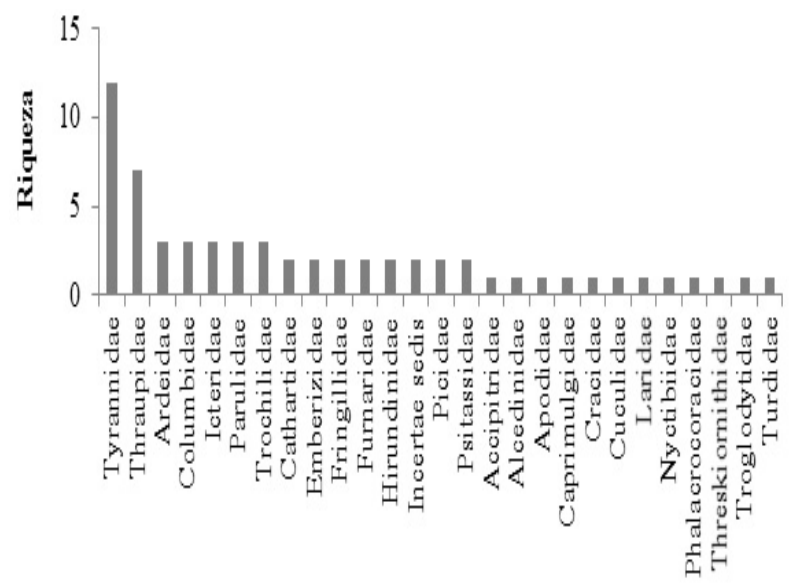

Figura 3. Distribución de la riqueza específica para las familias de la comunidad de aves presente en el campus de la Universidad de la Amazonia en Florencia, Caquetá, Colombia.
Tyrannidae (19,3\%) y Thraupidae (11,2\%) (Figura $3)$. Las especies con las mayores frecuencias de observación fueron Turdus ignobilis (7,1\%), Thraupis episcopus (6,6\%), Pitangus sulphuratus (6,3\%), Myiozetetes similis, Tyrannus melancholicus, y Ramphocelus carbo (5,5\%). Las frecuencias de observación de las tres primeras especies (T. ignobilis, T. episcopus y $P$. sulphuratus) representan $20,1 \%$ del total de los registros.

Las curvas de acumulación de especies indican que la representatividad del muestreo estuvo entre $82 \%$ y $87 \%$ (Figura 4 ); mientras que los índices de diversidad arrojaron valores importantes de rique$\mathrm{za}=10,35$, diversidad $=3,64$ y equidad $=0,88$, mientras que la dominancia $=0,04$ resultó ser muy baja.

Se registraron 10 gremios tróficos entre los cuales el más representativo fue el insectívoro (43,5\%); a este gremio pertenecen las familias Tyrannidae, Picidae y Furnaridae. El segundo gremio con mayor número de especies fue el piscívoro $(9,6 \%)$, donde se destacan las familias Ardeidae y Phalacrocoracidae. El resto de gremios tróficos estuvieron representados con una a cinco especies (Figura 5).

Se hallaron siete especies categorizadas en los apéndices II y III según la Convención sobre el Comercio Internacional de Especies Amenazadas de Fauna y Flora Silvestres (CITES). Entre las especies categorizadas en el apéndice II se encuentran: Rupornis magnirostris, Ara severus, Anthracothorax nigricollis, Amazilia fimbriata y Chlorostilbon mellisugus, estas especies no están amenazadas, sin embargo pueden llegar a estarlo si su comercio no se

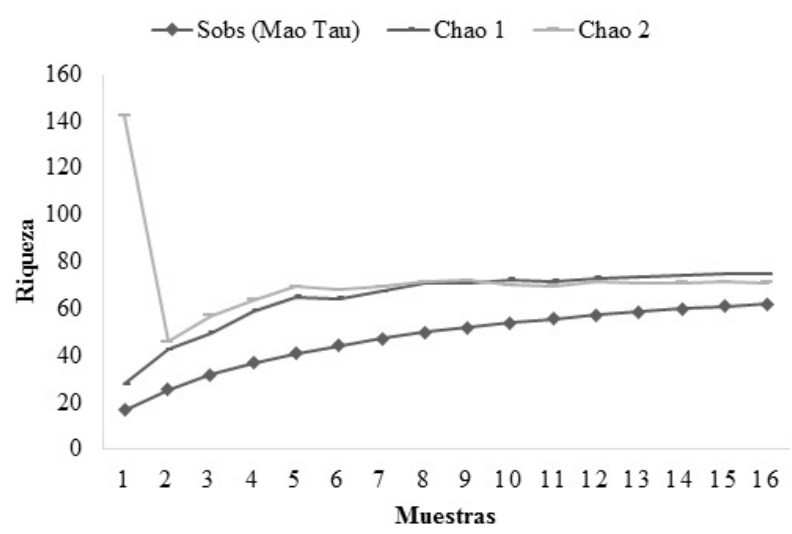

Figura 4. Curvas de acumulación de especies de la comunidad de aves presente en el campus de la Universidad de la Amazonia en Florencia, Caquetá, Colombia. 


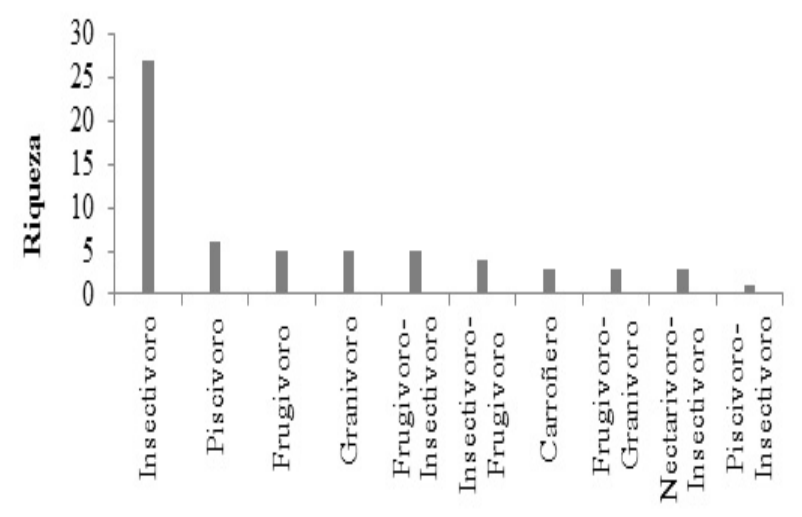

\section{Gremios}

Figura 5. Distribución de gremios tróficos de las aves presentes en el campus de la Universidad de la Amazonia en Florencia, Caquetá, Colombia.

controla (CITES 2015). Al apéndice III pertenecen las especies: Bubulcus ibis y Ardea alba, cuyo comercio es permitido siempre y cuando la autoridad del país de origen certifique que exportarlas no perjudica la supervivencia de las mismas (CITES 2015).

Se registraron seis especies de aves congregarías, las cuales ocupan lugares como playas de ríos, praderas y humedales, donde conforman colonias de anidación mientras descansan o hibernan. Entre estas especies se encuentran Phalacrocorax brasilianus, A. alba, B. ibis y Phimosus infuscatus. En cuanto a las aves migratorias se registraron trece especies, entre ellas algunas boreales como Setophaga striata, Setophaga petechia, Setophaga ruticilla y en menor cantidad australes como Tyrannus savana y Pygochelidon cyanoleuca (Tabla 1). Respecto a aves con algún grado de amenaza o endemismo no se tuvo ningún registro.

\section{Discusión}

A pesar de que se registró un número elevado de especies así como valores importantes en los índices de diversidad, los estimadores de riqueza sugieren que este estudio representa un primer acercamiento al conocimiento de la ornitofauna del campus y el área urbana del municipio de Florencia, aunque falta aproximadamente $20 \%$ de especies por registrar.

El predominio en los registros de las especies $T$. ignobilis, T. episcopus y P. sulphuratus, se puede relacionar con su facilidad de adaptación a ecosistemas intervenidos y con actividad antrópica (Sierra 2012), como es el caso del campus estudiado, donde la mayor parte de este se ha dedicado a actividades antrópicas. Estas especies no se consideraron como dominantes en este estudio, dados los valores arrojados por los índices de diversidad; sin embargo, esto podría cambiar con la adición del conjunto de especies que se espera sean registradas con la realización de más muestreos.

$\mathrm{La}$ alta diversidad que mostraron las familias Tyrannidae y Thraupidae coincide con los resultados obtenidos por Velásquez-Valencia (2009) en el Piedemonte Caqueteño, Marín (2005) en el campus de la Universidad del Quindío, Rivera (2006) en bosques secundarios de la ciudad de Cali y Muñoz et al. (2007) en el campus de la Universidad del Valle. Tal predominancia viene dada por las características del hábitat y la disponibilidad de alimento. En este sentido, el gremio trófico insectívoro al cual pertenecen especies de la familia Tyrannidae como, Pyrocephalus rubinus, Camptostoma obsoletum, Contopus cinereus, Myiodynastes maculatus, M. similis, T. savana, Tyrannus tyrannus y T. melancholicus, se vio favorecido por la presencia de áreas abiertas en el campus. Estas áreas por lo general presentan elementos como postes, cableado eléctrico, árboles y arbustos dispersos que brindan a estas especies sitios de percha donde la facilidad de observación es mayor al momento de realizar la captura del alimento (Orians 1969, Verea et al. 2000), en este caso de insectos, los cuales se encuentran disponibles en mayor cantidad hacia las horas de la tarde (Citelli 1993).

Los puntos que se destacan por presentar mayores áreas con espacios abiertos y donde estas especies aparecieron con mayor frecuencia son CFSC, CYSC, CFSP y EPSP. Por su parte los puntos TSP, ASP, ARSC y JBSC caracterizados por presentar una vegetación más densa, fueron más atractivos para especies de aves frugívoras en su mayoría de la familia Thraupidae. A pesar de la alta riqueza que presentó esta familia, el gremio frugívoro al cual pertenecen la gran mayoría de sus especies, no fue el segundo más diverso, en su lugar se ubicó el gremio de los piscívoros, cuya dominancia se puede relacionar con la presencia de ecosistemas hídricos (quebrada La Perdiz y río Hacha) en las zonas aledañas a ambas sedes del campus. Sin embargo, es de aclarar que en su mayoría estas especies no hicieron un uso directo del hábitat, sino, que se desplazaron con regularidad por el espacio aéreo del mismo y solo en algunas oca- 
Tabla 1. Listado taxonómico de la avifauna presente en el campus de la Universidad de la Amazonia en Florencia, Caquetá, Colombia

\begin{tabular}{|c|c|c|c|c|c|c|c|c|}
\hline Orden & Familia & Especie & $\begin{array}{l}\text { Frecuen- } \\
\quad \text { cia }\end{array}$ & $\begin{array}{l}\text { Gre- } \\
\text { mios }\end{array}$ & $\begin{array}{l}\text { Migra- } \\
\text { torias }\end{array}$ & Sede & $\begin{array}{l}\text { Congre- } \\
\text { garías }\end{array}$ & $\begin{array}{l}\text { CITES- } \\
\text { UICN }\end{array}$ \\
\hline Galliformes & Cracidae & Ortalis guttata & 1 & $\mathrm{~F}$ & & SC & & \\
\hline Suliformes & Phalacrocoracidae & Phalacrocorax brasilianus & 1 & $P$ & MB & SC & Co & \\
\hline \multirow[t]{4}{*}{ Pelecaniformes } & Ardeidae & Ardea alba & 3 & $\mathrm{P}$ & MB & SC & Co & III \\
\hline & & Bubulcus ibis & 2 & PI & MB & SC & Co & III \\
\hline & & Butorides striatus & 1 & $P$ & & SC & & \\
\hline & Threskiornithidae & Phimosus infuscatus & 7 & $P$ & & $\mathrm{CP}$ & Co & \\
\hline \multirow[t]{2}{*}{ Cathartiformes } & Cathartidae & Cathartes aura & 1 & $\mathrm{C}$ & MB & SP & Co & \\
\hline & & Coragyps atratus & 15 & C & & $\mathrm{CP}$ & & \\
\hline Accipitriformes & Accipitridae & Rupornis magnirostris & 5 & $\mathrm{C}$ & & $\mathrm{CP}$ & & II \\
\hline Charadriiformes & Laridae & Sternula superciliaris & 4 & $\mathrm{P}$ & & $\mathrm{CP}$ & Co & \\
\hline \multirow[t]{3}{*}{ Columbiformes } & Columbidae & Columba livia & 2 & FG & & SP & & \\
\hline & & Columbina talpacoti & 10 & FG & & $\mathrm{CP}$ & & \\
\hline & & Patagioenas cayennensis & 3 & $\mathrm{FG}$ & & SP & & \\
\hline \multirow[t]{2}{*}{ Psittaciformes } & Psitassidae & Amazona ochrocephala & 4 & $\mathrm{~F}$ & & SC & & \\
\hline & & Ara severus & 2 & $\mathrm{~F}$ & & SC & & II \\
\hline Cuculiformes & Cuculidae & Crotophaga ani & 3 & I & & $\mathrm{CP}$ & & \\
\hline \multirow[t]{2}{*}{ Caprimulgiformes } & Caprimulgidae & Nictidromus albicolis & 1 & I & & SC & & \\
\hline & Nyctibiidae & Nyctibius grandis & 1 & I & & SC & & \\
\hline \multirow[t]{4}{*}{ Apodiformes } & Apodidae & Tachornis squamata & 3 & I & & $\mathrm{CP}$ & & \\
\hline & Trochilidae & Amazilia fimbriata & 1 & $\mathrm{NI}$ & & SP & & II \\
\hline & & Anthracothorax nigricollis & 2 & $\mathrm{NI}$ & & SP & & II \\
\hline & & Chlorostilbon mellisugus & 2 & $\mathrm{NI}$ & & $\mathrm{CP}$ & & II \\
\hline Coraciiformes & Alcedinidae & Chloroceryle amazona & 4 & $P$ & & SC & & \\
\hline \multirow[t]{2}{*}{ Piciformes } & Picidae & Colaptes punctigula & 4 & I & & $\mathrm{CP}$ & & \\
\hline & & Picumnus lafresnayi & 1 & I & & SP & & \\
\hline \multirow{27}{*}{ Passeriformes } & Furnaridae & Xiphorhynchus guttatus & 3 & I & & SP & & \\
\hline & & Xiphorhynchus obsoletus & 2 & I & & $\mathrm{CP}$ & & \\
\hline & Tyrannidae & Camptostoma obsoletum & 6 & I & & $\mathrm{CP}$ & & \\
\hline & & Contopus cinereus & 6 & I & & $\mathrm{CP}$ & & \\
\hline & & Machetornis rixosa & 1 & I & & $\mathrm{CP}$ & & \\
\hline & & Myiodynastes maculatus & 3 & I & MA & SC & & \\
\hline & & Myiozetetes similis & 20 & I & & $\mathrm{CP}$ & & \\
\hline & & Pitangus sulphuratus & 23 & I & & $\mathrm{CP}$ & & \\
\hline & & Pyrocephalus rubinus & 7 & I & MA & SP & & \\
\hline & & Todirostrum chrysocrotaphum & 1 & I & & SC & & \\
\hline & & Todirostrum cinereum & 11 & I & & $\mathrm{CP}$ & & \\
\hline & & Tyrannus melancholicus & 20 & I & MA & $\mathrm{CP}$ & & \\
\hline & & Tyrannus savana & 3 & I & MA & $\mathrm{CP}$ & & \\
\hline & & Tyrannus tyrannus & 11 & I & MB & $\mathrm{CP}$ & & \\
\hline & Hirundinidae & Atticora fasciata & 1 & I & & SP & & \\
\hline & & Pygochelidon cyanoleuca & 2 & I & MA & $\mathrm{CP}$ & & \\
\hline & Troglodytidae & Troglodytes aedon & 18 & I & & $\mathrm{CP}$ & & \\
\hline & Turdidae & Turdus ignobilis & 26 & IF & & $\mathrm{CP}$ & & \\
\hline & Thraupidae & Ramphocelus carbo & 20 & $\mathrm{FI}$ & & $\mathrm{CP}$ & & \\
\hline & & Sicalis flaveola & 6 & G & & $\mathrm{CP}$ & & \\
\hline & & Sporophila minuta & 1 & G & & SC & & \\
\hline & & Sporophila murallae & 1 & $\mathrm{G}$ & & SC & & \\
\hline & & Thraupis episcopus & 24 & $\mathrm{FI}$ & & $\mathrm{CP}$ & & \\
\hline & & Thraupis palmarum & 5 & $\mathrm{FI}$ & & $\mathrm{CP}$ & & \\
\hline & & Volatinia jacarina & 7 & $\mathrm{G}$ & & $\mathrm{CP}$ & & \\
\hline & Incertae Sedis & Saltator coerulescens & 2 & $\mathrm{~F}$ & & SC & & \\
\hline & & Saltator maximus & 3 & $F$ & & $\mathrm{CP}$ & & \\
\hline
\end{tabular}


Tabla 1. Listado taxonómico de la avifauna presente en el campus de la Universidad de la Amazonia en Florencia, Caquetá, Colombia (continuación)

\begin{tabular}{|c|c|c|c|c|c|c|c|c|}
\hline Orden & Familia & Especie & $\begin{array}{l}\text { Frecuen- } \\
\quad \text { cia }\end{array}$ & $\begin{array}{l}\text { Gre- } \\
\text { mios }\end{array}$ & $\begin{array}{l}\text { Migra- } \\
\text { torias }\end{array}$ & Sede & $\begin{array}{l}\text { Congre- } \\
\text { garías }\end{array}$ & $\begin{array}{l}\text { CITES- } \\
\text { UICN }\end{array}$ \\
\hline & \multirow[t]{2}{*}{ Emberizidae } & Ammodramus aurifrons & 13 & G & & $\mathrm{CP}$ & & \\
\hline & & Arremonops conirostris & 2 & 1 & & SC & & \\
\hline & \multirow[t]{3}{*}{ Parulidae } & Setophaga petechia & 8 & I & MB & $\mathrm{CP}$ & & \\
\hline & & Setophaga ruticilla & 5 & 1 & MB & $\mathrm{CP}$ & & \\
\hline & & Setophaga striata & 5 & I & MB & $\mathrm{CP}$ & & \\
\hline & \multirow[t]{3}{*}{ Icteridae } & Icterus nigrogularis & 1 & IF & & SC & & \\
\hline & & Psarocolius angustifrons & 1 & IF & & $\mathrm{SP}$ & & \\
\hline & & Psarocolius decumanus & 9 & IF & & $\mathrm{CP}$ & & \\
\hline & \multirow[t]{2}{*}{ Fringillidae } & Euphonia chrysopasta & 1 & $\mathrm{FI}$ & & SC & & \\
\hline & & Euphonia laniirostris & 2 & $\mathrm{FI}$ & & $\mathrm{CP}$ & & \\
\hline
\end{tabular}

I: Insectívoro, P: Piscívoro, F: Frugívoro, Fl: Frugívoro-Insectívoro, IF: Insectívoro-Frugívoro, G: Granívoro, C: Carroñero, FG: Frugívoro-Granívoro, NI: Nectarivoro-Insectívoro, PI: Piscívoro-Insectívoro, MB: Migratoria boreal, MA: Migratoria austral, SC: Sede Centro, SP: Sede Porvenir, CP: Sede Centro y Porvenir, CITES I y II.

siones se observaron perchados algunos individuos de Butorides striatus y P. infuscatus.

Este trabajo fue realizado en un tiempo corto, que no permitió evidenciar los cambios que se pueden dar a lo largo del año, por tal razón, desarrollar estudios a largo plazo y con esfuerzos de muestreo más amplios, permitirá tener un conocimiento más preciso de las poblaciones de aves que habitan el campus e indirectamente el área urbana del municipio de Florencia y como varían a través del tiempo.

\section{Conclusiones}

La diversidad de aves registradas en el campus de la Universidad de la Amazonia sugiere que esta área representa una fuente importante de recursos como frutos, semillas, invertebrados, perchas y espacios de anidación, para las poblaciones de aves residentes y migratorias que habitan el área urbana del municipio de Florencia.

Es importante disminuir al máximo las perturbaciones que pudiesen generarse sobre las zonas verdes del campus, en especial las que prestan mayor complejidad estructural y diversidad vegetal como el jardín botánico (JBSC) o las áreas aledañas al auditorio (ASP), para así favorecer y mantener la oferta de recursos, porque estos parches de vegetación y demás áreas arboladas en el campus, favorecen la conectividad y la heterogeneidad de hábitats en la matriz urbana amortiguando así los efectos negativos de la urbanización sobre la avifauna.

Las especies que reportaron las mayores frecuen- cias no se consideraron como dominantes en este estudio, sin embargo, esto se lograría comprobar en el futuro una vez se tengan datos de estudios que incluyan las especies faltantes.

La presencia en el campus de un importante número de especies residentes, migratorias, congregarías y con comercio restringido, evidencia el alto valor ecológico del campus y su potencial como área de conservación de la ornitofauna urbana en el municipio de Florencia.

\section{Agradecimientos}

Este trabajo fue posible gracias a la colaboración del profesor Alexander Velásquez quien brindó su apoyo en el área de estadística; de igual forma queremos agradecer a Yardany Ramos, Javier E. García, David Sanín y Diego Carantón por la revisión del documento y sus sugerencias y finalmente un especial agradecimiento a Ángela Lugo por sus traducciones al idioma inglés.

\section{Literatura citada}

Angarita-Martínez I. 2002. Composición y estructura de la avifauna de la ciudad de Cali. (Trabajo de grado). Cali: Facultad de Ciencias Naturales y Exactas, Universidad del Valle; 48 pp.

Citelli LF. 1993. Importancia de la iluminación nocturna artificial en la alimentación de algunas especies de aves urbanas. (Trabajo de grado). Cali: Universidad del Valle; $57 \mathrm{pp}$.

CITES. 2015. The CITES Appendices. (En línea) [acceso 5 de febrero de 2015]. Disponible en: https://www.cites.org/ 
eng/app/index.php

Clergeau P, Jokimäki, J, Savard JPL. 2001. Are the urban birds communities influenced by the bird diversity of adjacent landscapes? Journal of Applied Ecology. 38: 1122-34.

Colwell RK, Chao A, Gotelli NJ, Lin S-Y, Mao CX, Chazdon RL, et al. 2012. Models and estimators linking individual-based and sample-based rarefaction, extrapolation, and comparison of assemblages. J Plant Ecol. 5 (1): 3-21.

Díaz-Cháux JT, Velásquez-Valencia A. 2009a. Aves de una laguna urbana en el Piedemonte Caqueteño colombiano. Momentos de Ciencia. 6 (1): 14-9.

Díaz-Cháux JT, Velásquez-Valencia A. 2009b. Uso de las coberturas vegetales y movilidad de aves semilleras en un paisaje urbano de Florencia (Caquetá, Colombia). Momentos de Ciencia. 6 (1): 70-9.

Halffter G. 1992. La diversidad biológica de Iberoamérica I. Xalapa: Instituto de Ecología, AC.

Hammer Ø, Harper DAT, Ryan PD. 2001. PAST: Paleontological statistics software package for education and data analysis. Palaeontologia Electronica. 4 (1): 9.

Hilty SL, Brown WL. 2001. Guía de las Aves de Colombia. The Plains: American Bird Conservancy (ABC); 1030 pp.

IDEAM. 2015. Características climatológicas de ciudades principales y municipios turísticos. (En línea) [acceso 5 de febrero]. Disponible en: http://www.ideam.gov.co

Marín Gómez OH. 2005. Avifauna del campus de la universidad del Quindío. Boletín SAO. 15 (2): 42-60.

McMullan M, Quevedo A, Thomas MD. 2011. Guía de campo de las aves de Colombia. Bogotá: Fundación Proaves.

Muñoz MC, Fierro-Calderón K, Rivera-Gutiérrez HF. 2007. Las aves del campus de la Universidad del Valle, una isla verde urbana en Cali, Colombia. Ornitol Colomb. 5: 5-20.

Murillo-Pacheco JI. 2005. Evaluación de la distribución y estado actual de los registros ornitológicos de los llanos orientales de Colombia. (Trabajo de grado). Pasto: Facultad de Ciencias Básicas y Matemáticas, Universidad de Nariño; $141 \mathrm{pp}$.

Naranjo LG, Amaya JD, Eusse-González D, Cifuentes-Sarmiento Y. 2012. Guía de las especies migratorias de la biodiversidad en Colombia. Vol. 1 Aves. Bogotá: Ministerio de Ambiente y Desarrollo Sostenible/WWF Colombia. 708 pp.

Naranjo LG, Estela F. 1999. Inventario de la avifauna de un área suburbana de la ciudad de Cali. Boletín SAO. 10: 11-27.

Orians GH. 1969. The number of bird species in some tropical forests. Ecology. 50 (5): 783-801.

Ortega-Álvarez R, MacGregor-Fors I. 2011. Dusting off the file: A review of knowledge on urban ornithology in Latin America. Landscape and Urban Planning. 101: 1-10. doi:10.1016/j.landurbplan.2010.12.020
Remsen JVJr, Areta JI, Cadena CD, Jaramillo A, Nores M, Pacheco JF, et al. 2015. A classification of the bird species of South America. (En línea) [acceso 5 de febrero]. Disponible en: http://www.museum.lsu.edu/ Remsen/ SACCBaseline.html

Renjifo LM, Franco-Maya AM, Amaya-Espinel JD, Kattan GH, López-Lanús B. 2002. Libro rojo de aves de Colombia. Bogotá: Instituto de Investigación de Recursos Biológicos Alexander von Humboldt y Ministerio del Medio Ambiente; 562 pp.

Restall R, Rodne C, Lentino M. 2007. Birds of northern South America: an identification guide. Vol. 1. Species accounts. New Haven: Yale University Press; 880 pp.

Rivera-Gutiérrez HF. 2006. Composición y estructura de una comunidad de aves en un área suburbana en el suroccidente colombiano. Ornitol Colomb. 4: 28-38.

Ruiz SL, Sánchez E, Tabares E, Prieto A, Arias JC, Gómez R, et al. (eds). 2007. Diversidad biológica y cultural del sur de la Amazonia colombiana. Bogotá: Corpoamazonia, Instituto Humboldt, SINCHI, UAESPNN; 636 pp.

Sierra Vásquez MA. 2012. Ciudad y fauna urbana. Un estudio de caso orientado al reconocimiento de la relación hombre, fauna y hábitat urbano en Medellín. (Trabajo de grado). Medellín: Facultad de Arquitectura, Universidad Nacional de Colombia; 66 pp.

Stiles FG, Skutch AF. 2003. Guía de aves de Costa Rica. $3^{\mathrm{a}}$ edición. Santo Domingo de Heredia: Editorial INBio; 571 pp.

Vargas Gutiérrez NP, Gómez JR, Velásquez Valencia A. 2005. Avifauna de la vereda Sebastopol, un área de bosques intervenido en el piedemonte caqueteño. Momentos de Ciencia 2 (1): 24-31.

Velásquez-Valencia A, Ricaurte LF, Lara F, Cruz EJ, Tenorio GA, Correa M. 2005. Lista anotada de las aves de los humedales de la parte alta del departamento de Caquetá. Memorias: Manejo de Fauna Silvestre en Amazonia y Latinoamérica; Iquitos, Perú. pp. 320-9.

Velásquez-Valencia A. 2009. Estructura de la comunidad de aves en sistemas de producción del Piedemonte Amazónico. Florencia: Facultad de Ciencias Naturales y Exactas, Universidad Nacional de Colombia; $51 \mathrm{pp}$.

Verea C, Fernández-Badillo A, Solórzano A. 2000. Variación en la composición de las comunidades de aves de sotobosque de dos bosques en el norte de Venezuela. Ornitol Neotrop. 11: 65-79.

Villarreal H, Álvarez M, Córdoba S, Escobar F, Fagua G, Gast F, et al. 2006. Manual de métodos para el desarrollo de inventarios de biodiversidad. $2^{\mathrm{a}}$ edición. Bogotá: Instituto de Investigación de Recursos Biológicos Alexander von Humboldt; 236 pp. 